\title{
VARIATION CHARACTERISTICS OF JUPITER'S HECTOMETRIC RADIATION DURING THE IOGENIC PLASMA ENHANCEMENT PERIOD
}

\author{
H. Misawa*, F. Tsuchiya*, T. Kimura ${ }^{\dagger}$, Y. Kasaba ${ }^{\ddagger}$ and A. Kumamoto ${ }^{\ddagger}$
}

\begin{abstract}
Around Jupiter's oppositions to Earth in 2014 and 2015, remote observations for Jupiter had been made intensively by the Hisaki satellite. In particular in the 2015 campaign period, sudden enhancement of Iogenic gas and plasma emissions was detected in the middle of January and the enhancement had lasted for more than two months [Yoneda et al., 2015, Icarus 261, 31-33]. This phenomenon would give a good opportunity to evaluate how the Iogenic plasma affects magnetosphere's variations.

We have analyzed Jupiter's hectometric radiations (HOM) for the period using the radio wave data of the WIND spacecraft placed around the L1 point of Earth. HOM is known to be one of the indicators reflecting Jupiter's global magnetospheric activities [e.g. Louarn et al., 2014, JGR 119, 4495-4511], and to have two components; i.e., one has some relations with solar wind variations ('solar wind HOM') and another one has almost no relation ('non solar wind HOM') [Nakagawa et al., 2000, Adv. Space Res. 26, 1541-1544]. In the presentation, we will introduce the following results from the preliminary analysis: 1) activity of (non-solar wind) HOM increased after the Iogenic plasma enhancement, 2) the enhancement is generally intermittent and quasi-periodic as if it reflected the occurrence of substorm like events in the magnetosphere, and 3) intensity variations of Jupiter's aurora and HOM are similar, but not always the same.
\end{abstract}

Acknowledgments. We greatly appreciate M. Kaiser and the WIND/WAVES team for providing the radio wave data through the WIND/WAVES home page.

\footnotetext{
* Planetary Plasma \& Atmospheric Research Center, Tohoku University, Sendai, Japan

${ }^{\dagger}$ RIKEN Nishina Center for Accelerator-Based Science, Tokyo, Japan

$\ddagger$ Graduate School of Science, Tohoku University, Sendai, Japan
} 
